\title{
LOCAL ESTIMATES FOR SUBSOLUTIONS AND SUPERSOLUTIONS OF OBLIQUE DERIVATIVE PROBLEMS FOR GENERAL SECOND ORDER ELLIPTIC EQUATIONS
}

\author{
GARY M. LIEBERMAN
}

Abstract. We consider solutions (and subsolutions and supersolutions) of the boundary value problem

$$
\begin{gathered}
a^{i j}(x, u, D u) D_{i j} u+a(x, u, D u)=0 \text { in } \Omega, \\
\beta^{i}(x) D_{i} u+\gamma(x) u=g(x) \text { on } \partial \Omega
\end{gathered}
$$

for a Lipschitz domain $\Omega$, a positive-definite matrix-valued function $\left[a^{i j}\right]$, and a vector field $\beta$ which points uniformly into $\Omega$. Without making any continuity assumptions on the known functions, we prove Harnack and Hölder estimates for $u$ near $\partial \Omega$. In addition we bound the $L^{\infty}$ norm of $u$ near $\partial \Omega$ in terms of an appropriate $L^{p}$ norm and the known functions. Our approach is based on that for the corresponding interior estimates of Trudinger.

This paper is concerned with analogs for solutions of oblique boundary value problems of the results in [10]. Our results will extend previous work of Lieberman and Trudinger [5] and of Nadirashvili [9]. Specifically we consider second order differential operators $Q$ of the form

$$
Q u=a^{i j}(x, u, D u) D_{i j} u+a(x, u, D u)
$$

in a domain $\Omega$ with Lipschitz boundary and first order operators $M$ of the form

$$
M u=\beta^{i}(x) D_{i} u(x)+\gamma(x) u(x)
$$

on $\partial \Omega$. The coefficients $a^{i j}, a$ are always assumed measurable, and the summation convention is observed. We also assume that $Q$ is elliptic at $u$, that is, the matrix $\left[a^{i j}(x, u, D u)\right]$ is everywhere positive definite with maximum eigenvalue $\Lambda$ and minimum eigenvalue $\lambda$. For our purposes, the obliqueness of $M$ is defined in a rather unusual fashion motivated by [3]. Set

$$
B\left(x_{0}, R\right)=\left\{x \in \mathbf{R}^{n}:\left|x-x_{0}\right|<R\right\} .
$$

A vector $\beta_{0}$ is oblique at $x_{0} \in \partial \Omega$ if there are a Lipschitz function $f$ and a positive constant $R$ such that

$$
\Omega \cap B\left(x_{0}, R\right)=\left\{x=\left(x^{\prime}, x^{n}\right) \in \mathbf{R}^{n}: x^{n}>f\left(x^{\prime}\right),|x|<R\right\}
$$

Received by the editors October 6,1986 .

1980 Mathematics Subject Classification (1985 Revision). Primary 35J65; Secondary 35J15. 
in an orthonormal coordinate system centered at $x_{0}$ for which $\beta_{0}$ is parallel to the positive $x^{n}$-axis. A vector field $\beta$ is oblique on a subset $S \subset \partial \Omega$ if $\beta\left(x_{0}\right)$ is oblique at $x_{0}$ for all $x_{0} \in S . M$ is oblique if the vector field $\beta$ is oblique. When $\partial \Omega \in C^{1}$, obliqueness of $\beta$ just means $\beta \cdot \nu>0$ for the inner unit normal $\nu$.

Under additional hypotheses on $Q, M$, and $\Omega$ (which will be spelled out later), we shall prove a weak Harnack inequality (Lemmas 2.1 and 2.2) and a local maximum principle (Theorems 3.3 and 3.4). As is well known the weak Harnack inequality provides a Hölder estimate (Corollary 2.3) and a strong minimum principal (Corollary 2.4) while the combination of the weak Harnack inequality with the local maximum principle gives a Harnack estimate (Corollary 3.5).

Similarly to [10], this work makes strong use of a Bakel'man-Aleksandrov-type maximum principle. We prove such a maximum principle in $\S 1$ by making some minor modifications of [6, Theorem 2.1]. Next the weak Harnack inequality is proved in $\$ 2$ by refining the choice of comparison function in [5, Lemma 2.1]; Hölder estimates also appear there. In $\S 3$, we prove the local maximum principle and a Harnack-type inequality.

Before proceeding, we point out that the definition of $M$ can be extended to functions which are not differentiable on $\partial \Omega$. We shall write $M u\left(x_{0}\right) \leqslant g\left(x_{0}\right)$ if

$\limsup _{t \rightarrow 0+}\left\{\frac{1}{t}\left[u\left(x_{0}+t \beta\left(x_{0}\right)\right)-u\left(x_{0}\right)\right]+\gamma\left(x_{0}+t \beta\left(x_{0}\right)\right) u\left(x_{0}+t \beta\left(x_{0}\right)\right)\right\} \leqslant g\left(x_{0}\right)$;

similarly $M u\left(x_{0}\right) \geqslant g\left(x_{0}\right)$ if $M\left(-u\left(x_{0}\right)\right) \leqslant-g\left(x_{0}\right)$ and $M u\left(x_{0}\right)=g\left(x_{0}\right)$ if $M u\left(x_{0}\right)$ $\leqslant g\left(x_{0}\right)$ and $M u\left(x_{0}\right) \geqslant g\left(x_{0}\right)$.

1. The global maximum principle. We begin with a variant of the Bakel'manAleksandrov maximum principle [1]. The variant is just a careful reworking of [6, Theorem 2.1], but it is important to spell out the technicalities for future reference.

We say that a vector $\beta_{0}$ points into a set $\Sigma$ at $x_{0} \in \partial \Sigma$ if there is a constant $t_{0}$ such that $x_{0}+t \beta_{0} \in \Sigma$ for all $t \in\left(0, t_{0}\right]$. Inward-pointing vector fields and differential operators are defined in the obvious way. Obviously obliqueness is a special case of this concept.

For a continuous function $u$ on $\Omega$, the upper contact set $\Gamma^{+}=\Gamma_{u}^{+}$is the set of all $y \in \Omega$ for which there exists $p \in \mathbf{R}^{n}$ such that

$$
u(x) \leqslant u(y)+p \cdot(x-y) \text { for all } x \in \Omega .
$$

The normal mapping $\chi$ is defined on subsets of $\Omega$ by

$$
\chi(\Sigma)=\left\{p \in \mathbf{R}^{n}:(1.1) \text { holds for some } y \in \Sigma\right\} .
$$

Also $\mathscr{D}=\operatorname{det}\left[a^{i j}\right]$.

Lemma 1.1 Suppose $\beta$ points into $\bar{\Omega}$ and suppose there are constants $\beta_{0} \geqslant 0, \gamma_{0}>0$ such that

$$
\gamma \leqslant-\gamma_{0},|\beta| \leqslant \beta_{0} \quad \text { on } \partial \Omega \text {. }
$$

Define the operator L by

$$
L u=a^{i j} D_{i j} u+b^{i} D_{i} u+c u
$$


and suppose there is a nonnegative constant $b_{0}$ such that

$$
\begin{aligned}
& \left\|b / \mathscr{D}^{1 / n}\right\|_{n ; \Omega} \leqslant b_{0}, \\
& c \leqslant 0 \quad \text { in } \Omega .
\end{aligned}
$$

If $u \in C^{0}(\bar{\Omega}) \cap C^{2}(\Omega)$ satisfies

$$
L u \geqslant f \text { in } \Omega, \quad M u \geqslant-g \text { on } \partial \Omega
$$

for some measurable $f$ and nonnegative constant $g$, and if $d=\operatorname{diam} \Omega$, then

$$
\sup _{\Omega} u \leqslant \frac{g}{\gamma_{0}}+C\left(n, b_{0}\right)\left(d+\frac{\beta_{0}}{\gamma_{0}}\right)\left\|\frac{f}{\mathscr{D}^{1 / n}}\right\|_{n ; \Gamma^{+}} .
$$

Proof. From the proof of the Bakel'man-Aleksandrov estimate in [2, Theorem 9.1], it suffices to show that

$$
\sup _{\Omega} u \leqslant \frac{g}{\gamma_{0}}+C(n)\left(d+\frac{\beta_{0}}{\gamma_{0}}\right)\left|\chi\left(\Gamma^{+}\right)\right|^{1 / n}
$$

To prove this estimate, we choose $R_{0}$ so that the ball of radius $R_{0}$ in $\mathbf{R}^{n}$ has volume equal to $\left|\chi\left(\Gamma^{+}\right)\right|$. If $R>R_{0}$, then there is a vector $p_{0} \in \mathbf{R}^{n} \backslash \chi\left(\Gamma^{+}\right)$with $\left|p_{0}\right|<R$. Consequently there is an affine function $W$ with $D w=p_{0}, w \geqslant u$ in $\bar{\Omega}$, and $w\left(x_{0}\right)=u\left(x_{0}\right)=u\left(x_{0}\right)$ for some $x_{0} \in \bar{\Omega}$. Because $p_{0} \notin \chi\left(\Gamma^{+}\right)$, it follows that $x_{0} \in \partial \Omega$. Moreover

$$
u\left(x_{0}\right) \leqslant \frac{1}{\gamma_{0}}\left(\beta \cdot D u\left(x_{0}\right)+g\right) \leqslant \frac{1}{\gamma_{0}}\left(\beta \cdot D w\left(x_{0}\right)+g\right) \leqslant \frac{1}{\gamma}\left(\beta_{0} R+g\right) .
$$

Because $u \leqslant w$, it follows that

$$
\sup _{\Omega} u \leqslant u\left(x_{0}\right)+R d \leqslant \frac{g}{\gamma_{0}}+\left(\frac{\beta_{0}}{\gamma_{0}}+d\right) R .
$$

By sending $R$ to $R_{0}$ in this inequality and then substituting for $R_{0}$, we obtain (1.6') and hence (1.6).

Note that there are no geometric restrictions on $\Omega$ (except for being bounded) in Lemma 1.1. Our proof of (1.6)' is essentially that of [6, Theorem 2.1] and Lemma 1.1 is a generalization of [6, Theorem 4.6]. The assumption $u \in W^{2, n}(\Omega) \cap C^{1}(\bar{\Omega})$ in [6] is recovered by a simple approximation argument. When $\beta$ and $\gamma$ are continuous, the regularity of $u$ can be further relaxed to $u \in W_{\text {loc }}^{2, n}(\Omega) \cap C^{0}(\bar{\Omega}), \beta \cdot D u \in C^{0}(\bar{\Omega})$ for some continuous extension of $\beta$. With this observation, we can infer the usual Bakel'man-Aleksandrov estimate [2, Theorem 9.1] as a special case of our lemma.

A related maximum estimate has been proved by Nadirashvili [9, Lemma 1.4]. The fundamental difference between his estimate and ours is that he assumes $c \leqslant 0$, $\gamma \leqslant 0$, and $c \not \equiv 0$. In proving his estimate, he also assumes that $a^{i j}$ is continuous and that $\partial \Omega \in C^{2}$. Since his estimates depend quantitatively on these assumptions, it is not clear how close they are to our (1.6).

2. Weak Harnack inequalities and Hölder estimates. Unlike the maximum estimate of Lemma 1.1, the weak Harnack inequality is affected strongly by the geometry of the domain. To avoid getting bogged down in technicalities, we consider some simple cases in detail. 
For the weak Harnack inequality we introduce some structure conditions. Let $\mu$ and $b_{0}$ be nonnegative constants and let $b, c, f$ be nonnegative measurable functions with $b \in L^{2 n}, c$ and $f$ in $L^{n}$. We assume that

$$
\begin{gathered}
a \geqslant-\lambda\left(b_{0}|p|^{2}+b|p|+c|z|+f\right), \\
\Lambda \leqslant \mu \lambda, \\
|\beta| \leqslant \mu, \gamma \leqslant 0 \quad \text { on } \partial \Omega .
\end{gathered}
$$

We also fix a point on $\partial \Omega$, which we take to be the origin, and we define $\Omega(R)=\Omega \cap\{|x|<R\}, \Sigma(R)=\partial \Omega \cap\{|x|<R\}$. Our other assumption is that there is a positive $R_{0}$ such that

$$
\beta^{n} \geqslant 1 \text { in } \Sigma\left(R_{0}\right) .
$$

LeMma 2.1. Set $\rho=1 / 16 n \mu$ and suppose that

$$
x^{n} \leqslant \frac{1}{2} \rho\left|x^{\prime}\right| \text { on } \Sigma\left(R_{0}\right) .
$$

Let $u \in C^{0}(\bar{\Omega}) \cap C^{2}(\Omega)$ and suppose also that there are nonnegative constants $m_{0}, g$ such that

$$
0 \leqslant u \leqslant m_{0}, \quad Q u \leqslant 0 \text { in } \Omega, \quad M u \leqslant g \text { on } \partial \Omega,
$$

the conditions $(2.1 \mathrm{a}, \mathrm{b})$ hold for $x \in \Omega, 0 \leqslant z \leqslant m_{0}, p \in \mathbf{R}^{n}$, and that (2.1c), (2.2) hold. If

$$
\begin{aligned}
& G(R)=\left\{x \in \Omega:\left|x^{\prime}\right|<R,-\rho R / 4<x^{n}<\rho R\right\}, \\
& G^{\prime}(R)=\left\{x \in \Omega:\left|x^{\prime}\right|<R, \rho R<x^{n}<3 \rho R / 2\right\},
\end{aligned}
$$

then there are positive constants $C, C_{1}, \kappa$ depending only on $n, \mu, b_{0} m_{0}$ such that

$$
0<R \leqslant \min \left\{\frac{C_{1}}{\left\|b^{2}+c\right\|_{n}}, \frac{R_{0}}{4}, \frac{1}{2\|\gamma\|_{\infty}}\right\}
$$

implies

$$
\left(\frac{1}{\left|G^{\prime}(2 R)\right|} \int_{G^{\prime}(2 R)} u^{\kappa}\right)^{1 / \kappa} \leqslant C\left[\inf _{G(R / 4)} u+R\|f\|_{n}+R g\right] .
$$

Proof. The hypotheses on $\Omega$ imply that $\operatorname{dist}\left(G^{\prime}(2 R), \partial \Omega\right) \geqslant C(n, \mu) R$. Therefore $[10$, Theorem 9] and a chaining argument give

$$
\left(\frac{1}{\left|G^{\prime}(2 R)\right|} \int_{G^{\prime}(2 R)} u^{\kappa}\right)^{1 / \kappa} \leqslant C\left[\inf _{G^{\prime}(2 R)} u+R\|f\|_{n}\right]
$$

for some positive $\kappa$ and $C$ depending on $n, \mu, b_{0} M_{0}$, and $C_{1}$. Hence we need only estimate $A=\inf _{G^{\prime}(2 R)} u$ in terms of the right-hand side of (2.7).

For this estimate, we replace $u$ by $\left(1-\exp \left(-b_{0} u\right) / b_{0}\right.$ to see that we may assume $b_{0}=0$. If we set $\mathbf{b}=b D u /|D u|$ (with the convention that $\mathbf{b}=0$ where $D u=0$ ) and

$$
L v=\frac{1}{\lambda} a^{i j} D_{i j} v+\mathbf{b}^{i} D_{i} v-c v,
$$


then $L u \leqslant f$ in $\Omega$. We now set $\alpha=16 / 35, G=G(2 R), G^{0}=\partial \Omega \cap \partial \Omega$, and introduce functions

$$
\begin{gathered}
w_{1}=2 \rho R-x^{n}, \quad w_{2}=2-\left(\frac{x^{n}}{2 \rho R}\right)^{2}-\frac{x^{n}}{2 \rho R}+\frac{\left|x^{\prime}\right|^{2}}{R^{2}}, \\
w=u+g w_{1}+\alpha A w_{2}-A .
\end{gathered}
$$

An easy calculation gives $w_{2} \geqslant 35 / 16=1 / \alpha$ if $x^{n}=-\rho R / 2$ and therefore

$$
\begin{gathered}
L w \leqslant b g+\alpha A b\left(\frac{3}{\rho}+4\right) \frac{1}{R}+c A+f \quad \text { in } G, \\
w \geqslant 0 \text { on } \partial \Omega \backslash G^{0} .
\end{gathered}
$$

Moreover on $G^{0}, M w \leqslant \alpha A M w_{2}+\|\gamma\|_{\infty} A$ and

$$
M w_{2} \leqslant \beta^{n}\left(\frac{x^{n}}{\rho R}+1\right) \frac{1}{2 \rho R}+\frac{4 \mu}{R} \leqslant-\frac{4}{R} .
$$

Therefore, because $R\|\gamma\|_{\infty} \leqslant 4 \alpha-1=29 / 35$,

$$
M w-w / R \leqslant 0 \quad \text { on } G^{0} \text {. }
$$

From Lemma 1.1 (with $\beta=0$ on $\partial G \backslash G^{0}$ ) we infer that

$$
-w \leqslant C(n, \mu) R\left(\|b\|_{n} g+\|f\|_{n}\right)+C(n, \mu) A\left(\|b\|_{n}+R\|c\|_{n}\right) \quad \text { in } G,
$$

with the $L^{n}$ norms taken over $G$. Assuming without loss of generality that $C_{1}<1$, it follows that $\alpha\|b\|_{n ; G}+R\|c\|_{n ; G} \leqslant C(n) C_{1}^{1 / 2}$. In $G(R / 4)$ we conclude that

$$
u+C\left(n, \mu, C_{1}\right) R\left(g+\|f\|_{n}\right) \geqslant A\left[1-C(n, \mu) C_{1}^{1 / 2}-17 \alpha / 8\right] .
$$

Because $17 \alpha / 8=34 / 35$, we can take $C_{1}=(1+70 C(n, \mu))^{-2}$ to infer that

$$
\inf _{G(R / 4)} u+C(n, \mu) R\left(\|f\|_{n}+g\right) \geqslant \frac{A}{70} .
$$

In conjugation with (2.8), this inequality gives (2.7).

If $\partial \Omega \in C^{1}$ and $\beta$ is oblique in the usual sense that $\beta \cdot \nu \geqslant 1$ for the inner normal $\nu$, Lemma 4.1 is applicable by taking $R_{0}$ small enough. Thus Lemma 4.1 includes [5, Lemma 2.1] as a special case. In this case, the restrictions on $R$ in terms of $b, c, \gamma$ can be eliminated by a chaining argument but then $C$ in (2.6) will depend also on $R\left\|b^{2}+c\right\|_{n}, R\|\gamma\|_{\infty}$.

Next we prove a weak Harnack inequality for a convex wedge.

LEMMA 2.2. Let $\tau$ be a positive constant and suppose

$$
\Omega\left(R_{0}\right)=\left\{x^{n}>\tau\left|x^{n-1}\right|,|x|<R_{0}\right\} .
$$

Let $u \in C^{0}(\bar{\Omega}) \cap C^{2}(\Omega)$ and suppose there are nonnegative constants $m_{0}$, guch that (2.4) holds. Suppose also that (2.1a,b) hold for $x \in \Omega, 0 \leqslant z \leqslant m_{0}, p \in R^{n}$ and that (2.1c), (2.2) hold. Suppose finally that

$$
\beta \cdot \nu \geqslant 1 \text { on the smooth part of } \Sigma\left(R_{0}\right) \text {. }
$$

If $\rho=1 /(4 n \mu), \tau^{*}=\rho^{2}\left(1+\tau^{2}\right)^{-1 / 2}, x^{\prime \prime}=\left(x^{1}, \ldots, x^{n-2}\right)$,

$$
G(R)=\left\{x \in \Omega: 0<x^{n}<\rho R,\left|x^{\prime \prime}\right|<R\right\},
$$


and

$(2.5 \mathrm{~b})^{\prime} \quad G^{\prime}(R)=\left\{x \in \Omega: \rho R<x^{n}<\frac{3}{2} r R, x^{n}>\tau\left|x^{n-1}\right|-\tau^{*} R \operatorname{sgn}\left(x^{n-1}\right)\right\}$,

then there are positive constants $C, C_{2}, \kappa$ depending only on $n, \mu, b_{0} m_{0}$ such that (2.7) is valid for

$$
0<R \leqslant \min \left\{\frac{C_{2}}{\left\|b^{2}+c\right\|_{n}}, \frac{R_{0}}{4}, \frac{1}{8\|\gamma\|_{\infty}}\right\} .
$$

Proof. Set $G^{\prime \prime}(R)=\left\{x \in \Omega:\left|x^{\prime \prime}\right|<R, x^{n}=\rho R\right\}$ and $A=\inf _{G^{\prime \prime}(R / 2)} \mu$. With $\alpha=\frac{1}{4}$ and $x^{\prime}$ replaced by $x^{\prime \prime}$ in the definition of $w_{2}$, the proof of Lemma 2.1 (see also [5, Lemma 2.1]) gives

$$
\inf _{G(R / 4)} u+C(n, \mu) R\left(g+\|f\|_{n}\right) \geqslant \frac{A}{5}
$$

provided $C_{2}$ is sufficiently small. Thus we need only estimate $\inf _{G^{\prime}(2 R)} u$ in terms of $\inf _{G^{\prime \prime}(R / 2)} u$. For this estimate, we invoke Lemma 2.1 at points in $\partial \Omega \cap \partial G^{\prime \prime}(R / 2)$ and we use a simple chaining argument to obtain

$$
\inf _{G^{\prime}(2 R)} u \leqslant C(n, \mu)\left[\inf _{G^{\prime \prime}(R / 2)} u+R g+R\|f\|_{n}\right] .
$$

Lemma 2.2 takes full advantage of the geometry of the convex wedge. By more careful application of the argument, we can prove a weak Harnack inequality if $\partial \Omega$ is locally the intersection of two $C^{1}$ hypersurfaces or if $\partial \Omega$ is locally a cone. In the latter case, we can show that

$$
\inf _{\Omega(R)} u \leqslant C(n, \mu)\left[\inf _{\Omega(3 R) \backslash \Omega(2 R)} u+R g+R\|f\|_{n}\right]
$$

(this inequality corresponds to (2.9)) very simply by taking

$$
w=u-A+g\left(2 R-x^{n}\right)+\frac{1}{4} A\left(2-x^{n} / 2 R\right) .
$$

More complex geometric configurations can be obtained by an expanded version of the argument in Lemma 2.2; for example, a weak Harnack inequality can be proved near a convex polyhedral boundary and for certain nonconvex domains as well. We shall not pursue this matter further.

Instead we state Hölder estimates for solutions of

$$
Q u=0 \text { in } \Omega, \quad M u=g \text { on } \partial \Omega
$$

under the structure conditions $(2.1 \mathrm{~b}, \mathrm{c})$ and

$$
|a(x, z, p)| \leqslant \lambda\left(b_{0}|p|^{2}+b|p|+c|z|+f\right) .
$$

COROLlaRy 2.3. Suppose there is a positive constant $R_{0}$ such that, for any $x_{0} \in \partial \Omega$, there is an orthonormal coordinate system center at $x_{0}$ in which (2.2) holds. Suppose also that (2.3) or $(2.3)^{\prime}$ holds and that if $(2.3)^{\prime}$ holds, so does $(2.2)^{\prime}$. If $u \in C^{0}(\bar{\Omega}) \cap$ $C^{2}(\Omega)$ is a solution of (2.10) with $m_{0}=\sup |u|$ and (2.11) being satisfied in $\Omega \times$ $\left[-m_{0}, m_{0}\right] \times \mathbf{R}^{n}$, then there is a positive constant $\alpha$ depending only on $n, \mu, b_{0} m_{0}$ such that $u \in C^{\alpha}(\bar{\Omega})$. Moreover

$$
|u|_{\alpha: \Omega} \leqslant C\left(n, \mu, b_{0} m_{0}, \operatorname{diam} \Omega, R_{0},\left\|b^{2}+c\right\|_{n},\|\gamma\|_{\infty}\right)\left(m_{0}+\|g\|_{\infty}+\|f\|_{n}\right) .
$$


Note that this form of the Hölder estimate follows because we can write

$$
\begin{gathered}
|a| \leqslant \lambda\left[\left(b_{0}+1 / m_{0}\right)|p|^{2}+\left(b^{2}+c\right) m_{0}+f\right], \\
|-\gamma u+g| \leqslant\|\gamma\|_{\infty} m_{0}+|g| .
\end{gathered}
$$

We can also write the Hölder estimate in the form

$$
\underset{\Omega(\sigma R)}{\operatorname{osc}} u \leqslant C \sigma^{\alpha}\left(m_{0}+\|g\|_{\infty}+\|f\|_{n}\right)
$$

for $\Omega(\sigma R)$ the intersection of $\Omega$ with a ball of radius $\sigma R$; see [10, Corollary 11].

Corollary 2.3 improves [5, Theorem 2.3] in relaxing the smoothness of $\partial \Omega$ and in allowing the coefficient $\Phi$ there to be an $L^{n}$ function rather than a constant. Our Hölder estimate also improves [9, Theorem 1.2] in relaxing the smoothness of $\partial \Omega$ and in removing the dependence of the constants on the modulus of continuity of the coefficients $a^{i j}$; the assumption $u \in W_{\text {loc }}^{2, n}(\Omega) \cap C^{1}(\bar{\Omega})$ in [9] is recovered in our case by approximation.

Another useful corollary of the weak Harnack inequality is a strong minimum principle (with the strong maximum principle obtained by modifying the hypotheses appropriately).

Corollary 2.4. Suppose $Q, M, \Omega$ satisfy the hypotheses of Corollary 2.3 with $f \equiv 0$ and (2.11) replaced by (2.1a). If $u \in C^{0}(\bar{\Omega}) \cap C^{2}(\Omega)$ satisfies

$$
\begin{gathered}
Q u \leqslant 0 \text { in } \Omega, \quad M u \leqslant 0 \text { on } \partial \Omega, \\
\inf _{\Omega} u \leqslant 0,
\end{gathered}
$$

then $u$ is a constant.

The corollary follows from Lemma 2.1 (or (2.2) and [10, Theorem 9] by the proof of [2, Theorem 8.19]. If $c \equiv 0$ and $\gamma \equiv 0$, then condition (2.14b) is not needed. Moreover if $c \not \equiv 0$ or $\gamma \not \equiv 0$, then clearly $u \equiv 0$. As a final consequence of the weak Harnack inequality, we have the following uniqueness result, which follows immediately from Corollary 2.4 .

Corollary 2.5. Let $R_{0}$, $\mu$ be positive constants. Suppose $a^{i j}, b^{i}$, c are measurable in $\Omega$ with $\Lambda \leqslant \mu \lambda, b^{i} / \lambda \in L^{2 n}, c / \lambda \in L^{n}$, and $c \leqslant 0$. Suppose $\beta^{i}$ and $\gamma$ are bounded on $\partial \Omega$ with $|\beta| \leqslant \mu$ and $\gamma \leqslant 0$ on $\partial \Omega$. Suppose also that $\beta$ points into $\bar{\Omega}$. If, at each $x_{0} \in \partial \Omega$, there is an orthonormal coordinate system centered at $x_{0}$ in which $\beta^{n} \geqslant 1$ in $\Sigma\left(R_{0}\right)$ and in which (2.3) or (2.3)' holds, then any $C^{0}(\bar{\Omega}) \cap C^{2}(\Omega)\left(\right.$ or $C^{1}(\bar{\Omega}) \cap$ $\left.W_{\mathrm{loc}}^{2, n}(\Omega)\right)$ solution of

$$
a^{i j} D_{i j} u+b^{i} D_{i} u+c u=0 \text { in } \Omega, \quad M u=0 \text { on } \partial \Omega
$$

is constant. Moreover if $\|c / \lambda\|_{n} \neq 0$ or $\gamma \neq \equiv$, then $u \equiv 0$.

Nadirashvili $[7,8]$ has proved the same result if $b^{i} / \lambda$ and $c / \lambda$ are bounded, $\beta$ is continuous and oblique, and $\Omega$ is Lipschitz. It would be of interest to see if the weak Harnack approach can be used in proving Nadirashvili's result, especially with the weaker hypotheses on the coefficients.

An application of Corollary 2.5 with discontinuous $\beta$ is given in [4]. 
3. The local maximum principle. In our proof of the local maximum principle, we shall use a function $\zeta$ with certain properties to be spelled out. This function $\zeta$ will play exactly the same role as $1-|x|^{2}$ does in [10].

Throughout this section, we set $M_{0}=\beta \cdot D$ and suppose that $0 \in \partial \Omega$. We then wish to construct a function $\zeta$ with the following properties:

$$
\begin{gathered}
\zeta \in C^{2}\left(\mathbf{R}^{n}\right), \quad \zeta \geqslant 0 \text { in } \mathbf{R}^{n}, \quad \zeta=0 \text { in }\{|x| \geqslant 1\}, \\
M_{0} \zeta \geqslant \zeta \quad \text { on } \partial \Omega \cap\{|x|<1\}, \\
\zeta(0)>0 .
\end{gathered}
$$

LEMMA 3.1. Let $\varepsilon$ and $\mu$ be positive constants and suppose

$$
\begin{gathered}
\left\{x^{n} \leqslant \frac{1-\varepsilon}{2 \mu}|x|,|x|<1\right\} \subset \Omega, \\
\beta^{n} \geqslant 1,|\beta| \leqslant \mu \quad \text { on } \partial \Omega \cap\{|x|<1\} .
\end{gathered}
$$

Then there is a function $\zeta$, determined only by $\varepsilon$ and $\mu$, satisfying (3.1).

Proof. For $\kappa$ and $R$ positive constants to be further specified, set

$$
\omega(x)=1+\frac{x^{n}}{R}-\kappa \frac{|x|^{2}}{R^{2}}, \quad \Omega^{*}=\{\omega>0\}, \quad \lambda=\frac{1-\varepsilon}{2 \mu} .
$$

On $\partial \Omega, \omega \leqslant 1+\lambda|x| / R-\kappa|x|^{2} / R^{2}$ so $\zeta$ will be positive only for $2 \kappa|x|<$ $R\left(\lambda+\left[\lambda^{2}+4 \kappa\right]^{1 / 2}\right)$. Assuming $\kappa$ has been chosen, we therefore choose $R$ so that $2 \kappa>R\left(\lambda+\left[\lambda^{2}+4 \kappa\right]^{1 / 2}\right)$. Hence

$$
M_{0} \omega=\beta^{n}-2 \kappa \beta \cdot x \geqslant \frac{1}{R}\left(1-\mu\left[\lambda+\left(\lambda^{2}+4 \kappa\right)^{1 / 2}\right]\right) \text { on } \Omega^{*} \cap \partial \Omega
$$

and therefore $M_{0} \omega \geqslant \varepsilon / 2 R$ on $\Omega^{*} \cap \partial \Omega$ if $\kappa$ is small enough. Since $\omega \leqslant 1+\lambda^{2} / 4 \kappa$ on $\partial \Omega$, there is a positive constant $\mu_{0}$ such that

$$
M_{0} \omega \geqslant \mu_{0} \omega \text { on } \Omega^{*} \cap \partial \Omega .
$$

The proof is completed by setting $\zeta=\max \{\omega, 0\}^{q}$ for $q$ sufficiently large.

If we wish to eliminate the hypothesis that $\Omega$ contains a large cone, a more subtle approach is needed. We shall only examine the case of a convex wedge.

LEMMA 3.2 Let $\tau_{1}, \tau_{2}, \mu$ be positive constants and set

$$
\begin{gathered}
\Omega=\left\{x \in \mathbf{R}^{n}: x^{n}>\max \left\{-\tau_{2} x^{n-1}, \tau_{1} x^{n-1}\right\},|x|<1\right\}, \\
\Sigma_{1}=\left\{x \in \partial \Omega: x^{n-1}>0\right\}, \quad \Sigma_{2}=\left\{x \in \partial \Omega: x^{n-1}<0\right\}, \\
\Sigma=\{x \in \partial \Omega:|x|<1\} .
\end{gathered}
$$

If

$$
\beta^{n} \geqslant 1 \text { and }|\beta| \leqslant \mu \text { on } \Sigma, \quad \beta \cdot \nu \geqslant 1 \text { on } \Sigma_{1} \cup \Sigma_{2},
$$

then there is a function $\zeta$ determined only by $\tau_{1}, \tau_{2}, \mu, n$ satisfying (3.1). 
Proof. Suppose first that $n=2$ and set

$$
\omega_{1}=1+x^{2}-4\left(x^{1}\right)^{2}, \quad \zeta_{1}=\max \left\{\omega_{1}, 0\right\}^{q} .
$$

If $q$ is large enough, there are positive constants $c_{0}, r_{0}, r_{1}$ with $r_{0}<r_{1}<1$ such that, on $\Sigma$,

$$
\begin{array}{ll}
M_{0} \zeta_{1} \geqslant \zeta_{1} & \text { if }|x| \leqslant r_{0}, \\
M_{0} \zeta_{1} \geqslant \zeta_{1}-c_{0} & \text { if } r_{0}<|x| \leqslant r_{1}, \\
\zeta_{1}=M_{0} \zeta_{1}=0 & \text { if }|x|>r_{1} .
\end{array}
$$

By adding together suitable functions from Lemma 3.2, we conclude that there is a $C^{2}$ function $\zeta_{2}$ with support in $\{|x|<1\}$ such that

$$
M_{0} \zeta_{2} \geqslant 2 \zeta_{2} \text { on } \Sigma, \quad \zeta_{2} \geqslant c_{0} \text { on } \Sigma \cap\left\{r_{0}<|x| \leqslant r_{1}\right\} \text {. }
$$

It follows that $\zeta=\zeta_{1}+\zeta_{2}$ is the desired function.

If $n>2$, we set $\zeta_{3}=\zeta_{1}+\zeta_{2}$ and

$$
\omega\left(x^{\prime \prime}, x^{n-1}, x^{n}\right)=\zeta_{3}\left(x^{n-1}, x^{n}\right)-\frac{1}{4} \zeta_{3}(0)-\frac{\zeta_{3}(0)}{4_{\mu}^{2}}\left|x^{\prime \prime}\right|^{2} .
$$

One easily checks that $M_{0} \omega \geqslant \omega$ on $\Sigma$; the desired $\zeta$ is just $\max \{\omega, 0\}^{2}$.

The local maximum principle follows easily with $\tau^{\prime}=\varepsilon$ if (3.2) holds and $\tau^{\prime}=\max \left\{\tau_{1}, \tau_{2}\right\}$ if $(3.3 \mathrm{a})$ holds

THEOREM 3.3. Suppose $\Omega$ and $\beta$ satisfy the hypotheses of Lemma 3.1 or Lemma 3.2. Suppose in addition that $Q$ satisfies the structure conditions

$$
\begin{gathered}
\Lambda \leqslant\left(a_{0}|\rho|^{N}+a_{1}\right) \mathscr{D}^{1 / n}, \\
a \leqslant\left(b_{0}|\rho|^{N+1}+b|p|+c z+f\right) \mathscr{D}^{1 / n}
\end{gathered}
$$

for all $(x, z, p) \in \Omega \times\left[0, m_{0}\right] \times \mathbf{R}^{n}$, where $m_{0}$ and $N$ are nonnegative constants, and $a_{0}, a_{1}, b_{0}, b, c, f$ are nonnegative functions with

$$
a_{0}, a_{1} \text { in } L^{r}(\Omega), \quad b_{0}, b_{1} \text { in } L^{q}(\Omega), \quad c, f \text { in } L^{n}(\Omega)
$$

for some $r \geqslant q>n$. Let $u \in C^{0}(\bar{\Omega}) \cap C^{2}(\Omega)$ satisfy

$$
\begin{gathered}
Q u \geqslant 0, u \leqslant m_{0} \quad \text { in } \Omega(1), \\
M u \geqslant-g \quad \text { on } \Sigma(1)
\end{gathered}
$$

for some nonnegative constant $g$, and let $R<1$. If

$$
h_{R}=\left(m_{0} / R\right)^{N}\left(a_{0}+R b_{0}\right)+a_{1}+b R,
$$

then for any $p>0$ and $\sigma \in(0,1)$, there is a constant $C$ depending only on $n, p, q, \mu$, $\tau / R\|\gamma\|_{\infty}, R^{-n / q}\left\|h_{R}\right\|_{q}, R\|c\|_{n}$ such that

$$
\sup _{\Omega(\sigma R)} u \leqslant C\left[\left(\frac{1}{|\Omega(R)|} \int_{\Omega(R)}\left(u_{+}\right)^{p}\right)^{1 / p}+R\|f\|_{n}\right] .
$$


Proof. Let $\varepsilon>0$. Assume without loss of generality that $R=1$, that $\|c\|_{n: \Omega(1)} \leqslant \varepsilon$, that $p \leqslant n$, and that $\sigma$ is so small that $\zeta>0$ in $\Omega(2 \sigma)$. Set

$$
s=n q(N+2) /(n-q) p, \quad v=\zeta^{s} u .
$$

On the contact set $\Gamma_{v}^{+}$of $v$ in $\Omega(1)$, we have (see [10, (26)])

$$
a^{i j} D_{i j} v \geqslant-\mathscr{D}^{1 / n}\left(C h_{1} \zeta^{-N-2} v+c v+f\right) .
$$

Moreover, on $\partial \Omega \cap\{u \geqslant 0\}$ we have

$$
M_{0} v=\zeta^{s} M_{0} u+s u \zeta^{s-1} M_{0} \zeta \geqslant-\gamma v+s v-g \geqslant v-g .
$$

If we redefine $\beta=0$ wherever $u \leqslant 0$, it follows that

$$
M_{0} v-v \geqslant-g \text { on } \partial \Omega(1) .
$$

Hence, from Lemma 1.1 we infer

$$
\sup _{\Omega(1)} v \leqslant C\left(\left\|h_{1} \zeta^{-N-2} v_{+}\right\|_{n}+\varepsilon \sup _{\Omega(1)} v+g+\|f\|_{n}\right)
$$

and then, for $\varepsilon$ small enough,

$$
\sup _{\Omega(1)} v \leqslant C\left(\left\|h_{1} \zeta^{-N-2} v_{+}\right\|_{n}+g+\|f\|_{n}\right) .
$$

The desired result in our special case follows from this inequality via Hölder's inequality and Young's inequality as in [10, p. 74]. The full result follows by a simple scaling and chaining argument.

A simple variant of the proof of Theorem 3.3 (see [10, Theorem 7]) gives an estimate independent of $m_{0}$.

THEOREM 3.4. Under the hypotheses of Theorem 3.3, if

$$
\begin{aligned}
& h_{R}^{\prime}=R^{-N}\left(a_{0}+b_{0}\right), \quad h_{R}^{\prime \prime}=a_{1}+b R, \\
& q^{*}=n q /(q-n), \quad p=(N+1) q^{*},
\end{aligned}
$$

then for any $\sigma \in(0,1)$, there is a constant $C=C\left(n, \sigma, N, \mu, \tau^{\prime}\right)$ for which

$$
\sup _{\Omega(\sigma R)} u \leqslant C\left[\frac{1}{R}\left(\left\|h_{R}^{\prime}\right\|_{q}\left\|\left(u_{+}\right)\right\|_{p}^{N+1}+\left\|h_{R}^{\prime \prime}\right\|_{q}\left\|u_{+}\right\|_{q^{*}}\right)+R g+R\|f\|_{n}\right] .
$$

Maximum estimates for solutions of

$$
Q u=0 \text { in } \Omega, \quad M u=g \text { on } \partial \Omega
$$

with $g$ bounded can be obtained easily; see [10, Corollary 8].

Harnack-type inequalities follow by combining Theorem 3.3 with Lemma 2.1 or Lemma 2.2.

Corollary 3.5. Let $Q, M, \Omega$ satisfy the hypotheses of Lemma 2.1 or 2.2 with (2.1a) replaced by (2.11) and let $u \in C^{0}(\bar{\Omega}) \cap C^{2}(\Omega)$ be a nonnegative solution of (3.11). If $y \in G^{\prime}(2 R)$ and if $\sigma$ is so small that the ball $B(y, 4 \sigma R)$ centered at $y$ with radius $4 \sigma R$ is contained in $G^{\prime}(2 R)$, then there is a constant $C$ depending only on $b_{0} m_{0}, n, \mu, \sigma$, $R_{0}\left\|b^{2}+c\right\|_{n}, R_{0}\|\gamma\|_{\infty}$ such that

$$
\sup _{B(y, \sigma R)} u \leqslant C\left(\inf _{G(R / 4)} u+R\|g\|_{\infty}+R\|f\|_{n}\right) .
$$




\section{REFERENCES}

1. Ya. Bakel'man, The Dirichlet problem for equations of Monge-Ampère type and their n-dimensional analogs, Dokl. Akad. Nauk SSSR 126 (1959), 923-926. (Russian)

2. D. Gilbarg and N. S. Trudinger, Elliptic partial differential equations of second order, 2nd ed., Springer-Verlag, Berlin and New York, 1983.

3. G. M. Lieberman, Oblique derivative problems in Lipschitz domains. I. Continuous boundary data, Boll. Un. Mat. Ital. (to appear).

4. , Oblique derivative problems in Lipschitz domains. II. Discontinuous boundary data (to appear).

5. G. M. Lieberman and N. S. Trudinger, Nonlinear oblique boundary value problems for fully nonlinear elliptic equations, Trans. Amer. Math. Soc. 295 (1986), 509-546.

6. P.-L. Lions, N. S. Trudinger, and J. I. E. Urbas, The Neumann problem for equations of Monge-Ampère type, Comm. Pure Appl. Math. 39 (1986), 539-563.

7. N. S. Nadirashvili, A lemma on the inner derivative, and the uniqueness of the solution of the second boundary value problem for second order elliptic equations, Dokl. Akad. Nauk SSSR 261 (1981), 804-808; English transl., Soviet Math. Dokl. 24 (1981), 598-601.

8. , On the question of the uniqueness of the solution of the second boundary value problem for second order elliptic equations, Mat. Sb. (N.S.) 122 (164) (1983), 341-359; English transl., Math. USSR-Sb. 50 (1985), 325-341.

9. On a problem with oblique derivative, Mat. Sb. (N.S.) 127 (169) (1985), 395-416. (Russian)

10. N. S. Trudinger, Local estimates for subsolutions and supersolutions of general second order elliptic quasilinear equations, Invent. Math. 61 (1980), 67-79.

Department of Mathematics, Iowa State University, Ames, Iowa 50011 